\title{
Osa-miR162a fine-tunes rice resistance to Magnaporthe oryzae and Yield
}

\author{
Xu-Pu Li ${ }^{1,2}$, Xiao-Chun Ma ${ }^{1,2}$, He Wang ${ }^{1,2}$, Yong Zhu ${ }^{1,2}$, Xin-Xian Liu ${ }^{1,2}$, Ting-Ting Li, ${ }^{1,2}$, Ya-Ping Zheng ${ }^{1,2}$, \\ Ji-Qun Zhao ${ }^{1,2}$, Ji-Wei Zhang ${ }^{1,2}$, Yan-Yan Huang ${ }^{1,2}$, Mei Pu ${ }^{1,2}$, Hui Feng ${ }^{1,2}$, Jing Fan ${ }^{1,2}$, Yan $\mathrm{Li}^{1,2^{*}}$ (D) and \\ Wen-Ming Wang ${ }^{1,2^{*}}$
}

\begin{abstract}
MicroRNAs (miRNAs) play essential roles in rice immunity against Magnaporthe oryzae, the causative agent of rice blast disease. Here we demonstrate that Osa-miR162a fine-tunes rice immunity against M. oryzae and yield traits. Overexpression of Osa-miR162a enhances rice resistance to $M$. oryzae accompanying enhanced induction of defense-related genes and accumulation of hydrogen peroxide $\left(\mathrm{H}_{2} \mathrm{O}_{2}\right)$. In contrast, blocking Osa-miR162 by overexpressing a target mimic of Osa-miR162a enhances susceptibility to blast fungus associating with compromised induction of defense-related gene expression and $\mathrm{H}_{2} \mathrm{O}_{2}$ accumulation. Moreover, the transgenic lines overexpressing Osa-miR162a display decreased seed setting rate resulting in slight reduced yield per plant, whereas the transgenic lines blocking Osa-miR162 show an increased number of grains per panicle, resulting in increased yield per plant. Altered accumulation of Osa-miR162 had a limited impact on the expression of rice Dicer-like 1 (OsDCL1) in these transgenic lines showing normal gross morphology, and silencing of OSDCL1 led to enhanced resistance to blast fungus similar to that caused by overexpression of Osa-miR162a, suggesting the involvement of OSDCL1 in Osa-miR162a-regulated resistance. Together, our results indicate that Osa-miR162a is involved in rice immunity against $M$. oryzae and fine-tunes resistance and yield.
\end{abstract}

Keywords: Osa-miR162, OsDCL1, Blast disease resistance, Yield traits, Hydrogen peroxide, Defense-related gene

\section{Background}

Plants mount a multi-layered immune system in fighting against the invasion of pathogens, and small RNAs are essential regulators in this process (Katiyar-Agarwal and Jin 2010; Weiberg et al. 2014). miRNAs are a subset of non-coding small RNAs of 20-24 nucleotides (nt) and play vital roles in the regulation of gene expression either by chromatin methyl modification, mRNA cleavage, or translational inhibition ( $\mathrm{Yu}$ et al. 2017). In plants, miRNAs are transcribed from miRNA genes and subsequently cleaved by the endoribonucleases DCL proteins, and processed into approximately 20-24 nt miRNAs (Kurihara and Watanabe 2004). Therefore, DCL1 plays

\footnotetext{
*Correspondence: jiazaihy@163.com; j316wenmingwang@sicau.edu.cn 'State Key Laboratory of Crop Gene Exploration and Utilization in Southwest China, Sichuan Agricultural University, Chengdu, China Full list of author information is available at the end of the article
}

important roles in the formation of mature miRNAs. In turn, the expression of $D C L 1$ is regulated by miR162 (Tijsterman and Plasterk 2004; Xie et al. 2004; Wu et al. 2009; Zhou et al. 2010).

miR162 is involved in multiple abiotic stress responses in plants. For example, in Arabidopsis, miR162 was induced by $\mathrm{ABA}$ treatment to improve adaptation to drought stress through suppressing Trehalase precursor 1 (OsTRE1) (Tian et al. 2015). In maize, miR162 was responsive to salt stress with enhanced accumulation at $0.5 \mathrm{~h}$ post-treatment of salt, whereas decreased at 5 and $24 \mathrm{~h}$ post-treatment (Ding et al. 2009). In switchgrass (Panicum virgatum), the accumulation of miR162 was significantly changed under drought stress (Sun et al. 2012). In cotton (Gossypium hirsutum L.), miR162 was responsive to salinity (Salih et al. 2018). Besides, miR162 also participates in biotic stress responses. For example, 
virus suppressors, such as Tombusvirus p19 and Cucumovirus 2b, had a binding preference for miR162 to counteract host antiviral RNA silencing, a plant immune response during viral infection initiation (Pertermann et al. 2018).

In Arabidopsis and rice, miR162 suppresses the expression of DCL1, which plays essential roles in miRNA biogenesis and metabolism (Tijsterman and Plasterk 2004; Xie et al. 2004; Zhou et al. 2010). In Arabidopsis, the loss-of-function mutants of AtDCL1 showed multiple altered phenotypes associating with the reduction of miRNA levels (Park et al. 2002). In rice, strong loss of function of OsDCL1 led to developmental arrest at the seedling stage. In contrast, weak loss of function of OsDCL1 resulted in pleiotropic developmental defects, including dark green, dwarfism, and different leaves and root phenotypes in comparison with control (Liu et al. 2005). Besides the roles in development, DCL1 also regulates resistance against pathogens. For example, AtDCL1 was required for miR393-mediated antibacterial defenses (Navarro et al. 2006). The silence of OsDCL1 enhanced resistance to the virulent Magnaporthe oryzae strains with enhanced induction of defense responses, including hydrogen peroxide accumulation and cell death at the infected sites (Zhang et al. 2015). In contrast, activation of OsDCL1a enhances susceptibility to fungal pathogens Fusarium fujikuroi and M. oryzae accompanying with compromised pathogen-inducible defense responses, and impacts miRNA network (Salvador-Guirao et al. 2019). These results indicate that OsDCL1 regulates rice growth and immunity against $M$. oryzae. Although Osa-miR162 suppresses the expression of OsDCL1 (Zhou et al. 2010), the effect of Osa-miR162 on rice blast resistance and yield is unclear. It is also unknown whether there are other target genes of OsamiR162 involved in these processes.

In rice, the Osa-miR162 family has two isoforms, namely Osa-miR162a and Osa-miR162b. Previously, we found that Osa-miR162a was responsive to M. oryzae in both susceptible and resistance accessions ( $\mathrm{Li}$ et al. 2014). Here we showed that Osa-miR162 fine-tuned rice growth and blast disease resistance. Our data demonstrated that overexpression of Osa-miR162a led to enhanced resistance but penalized yield, whereas blocking Osa-miR162 via a target mimic resulted in suppressed resistance but increased yield. In addition, OsDCL1 is involved in miR162-mediated resistance against $M$. oryzae.

\section{Results}

Osa-miR162a Is Differentially Responsive to M. oryzae in Susceptible and Resistant Accessions

To explore the involvement of Osa-miR162a in rice blast resistance, we examined the amounts of Osa-miR162a in a highly susceptible accession Lijiang xin Tuan Heigu
(LTH) and a resistant accession IRBLKm-Ts. LTH is sensitive to over 1300 regional isolates of $M$. oryzae worldwide and often used as a susceptible reference in blast disease assay (Lin et al. 2001). IRBLKm-Ts carries a resistance $(R)$ locus $P i-k m$ and exhibits resistance to $M$. oryzae isolates carrying Avr-Pikm (Tsumematsu et al. 2000). We identified the disease phenotype of LTH and IRBLkm-Ts by spray-inoculation of $M$. oryzae strain Guy11. LTH showed susceptible phenotype with serious disease lesions, whereas IRBLkm-Ts showed resistant phenotype with few small lesions (Fig. 1a). LTH displayed unchanged or decreased Osa-miR162a abundance following the inoculation of Guy11 in comparison with mock samples. In contrast, IRBLKm-Ts showed reduced accumulation at 12 and $48 \mathrm{hpi}$ but increased at $24 \mathrm{hpi}$ (Fig. 1b), indicating Osa-miR162a was involved in riceM. oryzae interaction.

\section{Overexpression of Osa-miR162a Enhances Rice Resistance to M. oryzae}

To explore the roles of Osa-miR162a in rice blast resistance, we constructed transgenic lines overexpressing Osa-miR162a (OX162) under Nipponbare (NPB) background. We got over 20 transgenic lines displaying increased amounts of mature Osa-miR162a in comparison with NPB control. We selected two lines showing moderate accumulation of Osa-miR162a and producing seeds, namely OX162-11 and OX162-24, for subsequent study (Fig. 2a). We then examined the blast disease phenotype of these lines following the punch-inoculation of two virulent strains, namely GZ8 and 97-27-2. GZ8 was an enhanced Green Fluorescence Protein (GFP)-tagged strain Zhong 810-14, which was derived from the paddy yard in China, and 97-27-2 was a strain derived from the paddy yard in Sichuan Province, China. OX162 lines displayed enhanced resistance with smaller disease lesions than control (Fig. 2b). Consistently, the fungal biomass in OX162 was significantly less than that in control (Fig. 2c). Then we examined the disease phenotype of OX162 by spray-inoculation of GZ8 and got the results similar to that by punch-inoculation (Additional file 1: Figure S1). Moreover, we also identified the resistance of OX162 against four M. oryzae strains isolated from paddy yard in Sichuan Province, China. Similarly, OX162 displayed smaller disease lesions than control (Additional file 2: Figure S2). These results indicate that overexpression of OsamiR162a enhances rice blast resistance.

To understand how OX162 lines suppress fungal growth, we observed the infection process of GZ8 in leaf sheath. At $24 \mathrm{hpi}$, more than $55 \%$ of spores formed appressoria, and quite a few of which formed invasive hyphae in sheath cells of WT. However, in OX162, less 

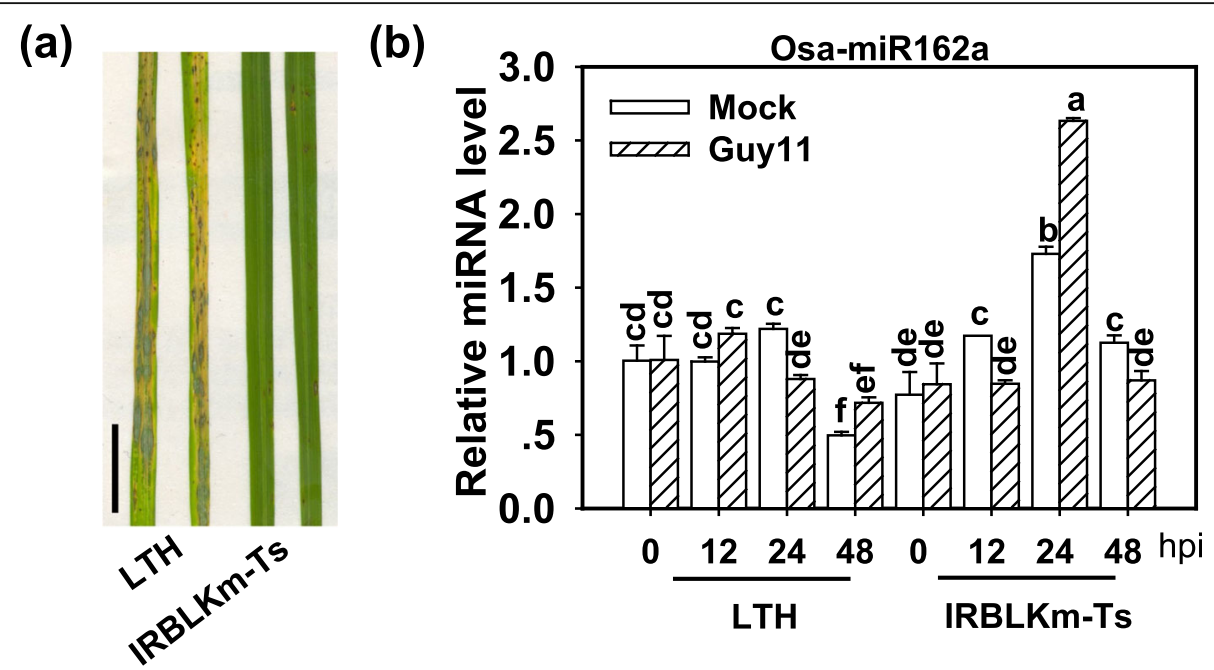

Fig. 1 Osa-miR162a is differentially responsive to Magnaporthe oryzae in the susceptible and resistance accessions. a Blast disease phenotype on leaves of susceptible accession LTH and resistant accession IRBLKm-Ts following spray-inoculation of Magnaporthe oryzae Guy $11\left(1 \times 10^{5}\right.$ spore/ $\mathrm{ml}$ concentration) at 5 days post-inoculation (dpi). Scale bar $=1 \mathrm{~cm}$. b Accumulation of Osa-miR162a in indicated accessions with or without Guy11 infection. Total RNA was used to carry out reverse-transcription (RT) with an Osa-miR162 specific stem-loop RT primers (Additional file 4: Table S1), and the RT product was subsequently used as a template for quantitative polymerase chain reaction (q-PCR) to detect the amounts of Osa-miR162. snRNA U6 served as an internal reference. Error bars indicate SD. Different letters above the bars indicate a significant difference $(P<$ $0.05)$ as determined by a one-way ANOVA analysis. Similar results were obtained in at least two independent experiments

than $50 \%$ of spores developed appressoria, and no invasive hyphae were observed (Fig. 2d). At 36 hpi, more than $50 \%$ of the spores started to infect the neighbor cells near the local infected cell of WT, whereas less than $20 \%$ of the spores invaded in the neighbor cells of OX162 (Fig. 2d). The quantification assay confirmed that overexpression of Osa-miR162a delayed the infection progress of blast fungus (Fig. 2e).

To understand why overexpression of Osa-miR162a delayed the infection of $M$. oryzae, we examined immune responses, such as $\mathrm{H}_{2} \mathrm{O}_{2}$ accumulation and induction of defense-related genes in OX162 and control. OX162 showed more $\mathrm{H}_{2} \mathrm{O}_{2}$ accumulation in the leaf cells around the infected sites than NPB following GZ8 inoculation (Fig. 3a). Four defense-related genes, namely ENT-KA RENE SYNTHASE 4 (KS4), NAC DOMAINCONTAINING PROTEIN 4 (NAC4), PATHOGENESISRELATE GENG 1A (PR1a), and PR10b, were examined in OX162 and NPB. KS4 is an early-induced basal defense-related gene (Park et al. 2012). NAC4 is involved in plant cell death and highly expressed in a lesion mimic mutant spl11 at the lesion forming period (Yin et al. 2000). PR1a and PR10b are pathogenesis-related genes (Yamaguchi et al. 2013). The induction of KS4 was enhanced to higher levels in OX162 than in NPB control at 12, 24 and $48 \mathrm{hpi}$, and NAC4 was enhanced to higher levels in OX162 than in NPB control at $12 \mathrm{hpi}$ and subsequently decreased at $24 \mathrm{hpi}$, then enhanced at 48 hpi again (Fig. 3b). Similarly, the expression of PR1a and $P R 10 b$ was significantly induced to higher levels at
12 and 48 hpi than that in NPB control (Fig. 3b). These data indicate that overexpression of Osa-miR162a enhanced the immune responses triggered by $M$. oryzae.

\section{Blocking Osa-miR162a Leads to Increased Blast Disease Susceptibility}

To further confirm the roles of Osa-miR162a in rice immunity against blast fungus, we constructed the transgenic lines overexpressing a target mimic of OsamiR162a (MIM162). MIM162 contained a sequence reversely complementary to Osa-miR162a with three nucleotides insertion between 10 to 11 nucleotide sites. Therefore, the target mimic acted as a sponge to absorb Osa-miR162 and block its binding to target genes (Additional file 3: Figure S3). We then examined the amounts of Osa-miR162a in MIM162. MIM162 displayed significantly less accumulation of Osa-miR162a than NPB control (Fig. 4a). Next, we conducted a disease assay on MIM162. As expected, MIM162 showed enhanced susceptibility to M. oryzae strain 97-27-2 and GZ8 with larger disease lesions following punch- or spray-inoculation (Fig. 4b and c, and Additional file 1: Figure S1). We also examined the disease phenotypes of MIM162 against four $M$. oryzae strains derived from the paddy yards by punch-inoculation. Similarly, MIM162 displayed larger disease lesions and supported more fungal growth than control (Additional file 2: Figure S2).

Moreover, the infection process of GZ8 in MIM162 was faster than that in control. At $24 \mathrm{hpi}$, more than $70 \%$ of spores formed appressoria or invasive hyphae in 

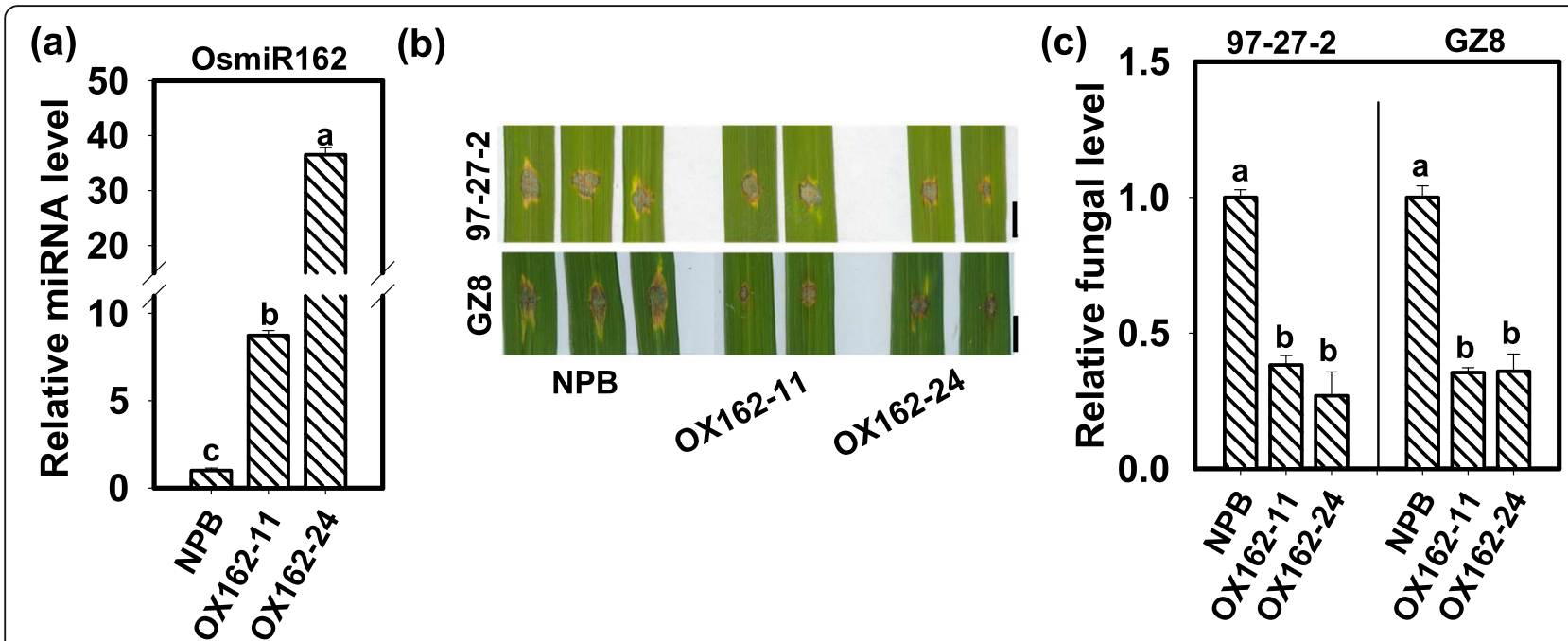

(d)
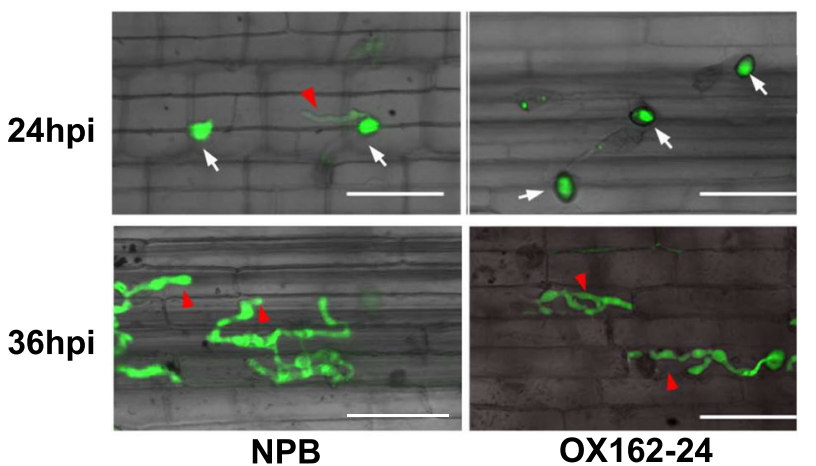

(e)

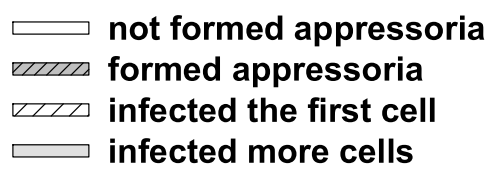

OX162-24 NPB OX162-24 NPB
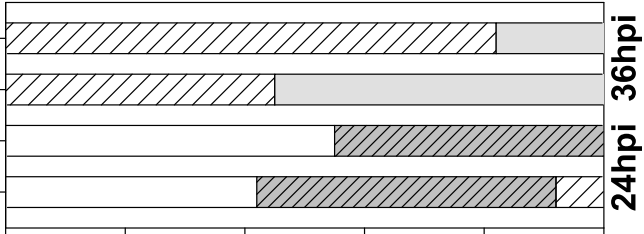

0

\section{$\begin{array}{llll}40 & 60 & 80 & 100\end{array}$ Percentage (\%)}

Fig. 2 Overexpression of Osa-miR162a enhances rice resistance to Magnaporthe oryzae. a Accumulation of Osa-miR162a in transgenic lines harboring 35S: Osa-miR162a (OX162). Total RNA was used to carry out reverse-transcription with an Osa-miR162 specific stem-loop RT primers (Additional file 4: Table S1), and the RT product was subsequently used as a template for quantitative polymerase chain reaction (q-PCR) to detect the amounts of Osa-miR162. snRNA U6 served as an internal reference. $\mathbf{b}$ Blast disease phenotypes on leaves at 5 days post-inoculation of $M$. oryzae strain GZ8 and 97-27-2, respectively. Bar $=5 \mathrm{~mm}$. c Relative fungal biomass of GZ8 and 97-27-2 on NPB and OX162. The relative fungal biomass was measured by using the ratio of DNA level of $M$. oryzae Pot2 gene against the rice genomic ubiquitin DNA level. $\mathbf{d}$ Invasion process of M. oryzae strain GZ8 at 24 and $36 \mathrm{~h}$ post-inoculation (hpi) on sheath cells of indicated lines. Bars $=25 \mu \mathrm{m}$. The white arrows indicate appressoria formed from conidia, and the red arrowheads indicate invasive hypha in rice sheath cells. e Quantification analysis on infection process. Over 200 conidia in each line were analyzed. For $(\mathbf{a})$ and $(\mathbf{c})$, Error bars indicate SD $(n=3)$. Different letters above the bars indicate significant differences $(P<0.05)$ as determined by One-way ANOVA analysis. Similar results were obtained in at least two

independent experiments

MIM162, in comparison with less than $60 \%$ of that in NPB control (Fig. 4d and e). At $36 \mathrm{hpi}$, more than $75 \%$ of spores invaded into the neighbor cells in MIM162, in comparison with less than $60 \%$ of that in NPB (Fig. $4 \mathrm{~d}$ and e). These results indicate that blocking Osa-miR162 facilitates the invasion of $M$. oryzae.

We also examined the defense responses in MIM162. NPB plant showed visible $\mathrm{H}_{2} \mathrm{O}_{2}$ accumulation at the infected sites of leaves and cells following GZ8 infection, whereas MIM162 displayed little $\mathrm{H}_{2} \mathrm{O}_{2}$ amounts at the invasive sites (Fig. 5a). Consistently, the induction of defense-related genes, KS4, NAC4, PR1a, and PR10b, was lower in MIM162 than that in NPB (Fig. 5b). These data indicate that blocking Osa-miR162 compromises immune responses induced by $M$. oryzae.

\section{Osa-miR162a Regulates Rice Yield Traits}

We found that Osa-miR162a also controlled rice agronomic traits. Both OX162 and MIM162 exhibited comparable phenotypes with WT, including gross morphology, panicle number per plant, and panicle length (Fig. 6a and Table 1). However, OX162 showed narrower seeds, resulting in lower seed weight than control, and significantly lower 
(a)

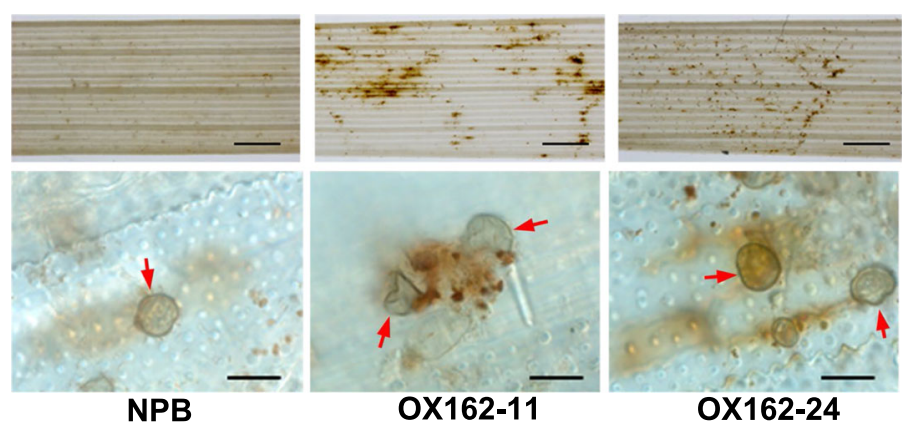

(b)
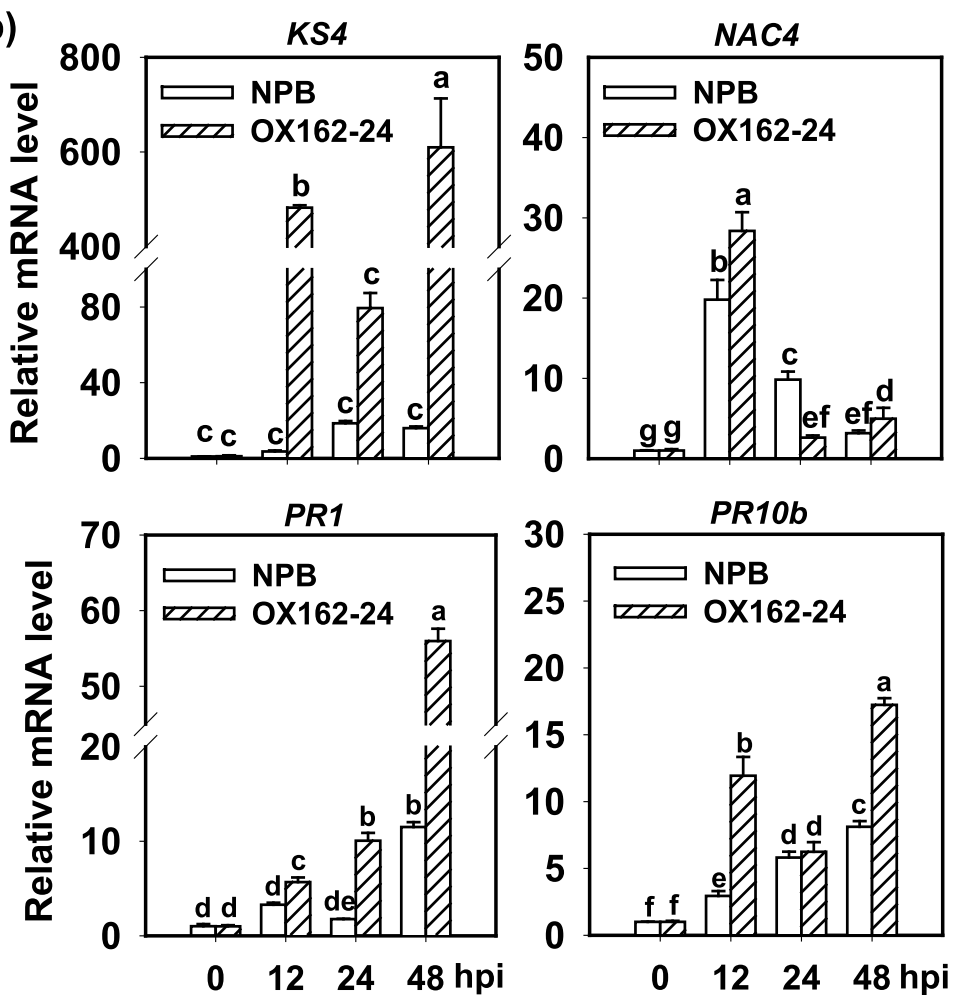

Fig. $\mathbf{3}$ Osa-miR162a enhances the induction of disease-related defense responses. a Hydrogen peroxide $\left(\mathrm{H}_{2} \mathrm{O}_{2}\right)$ accumulation in wild type (Nipponbare, NPB) and OX162 at $48 \mathrm{~h}$ post-inoculation (hpi) of M. oryzae strain GZ8. The intensity of brown indicates the amounts of $\mathrm{H}_{2} \mathrm{O}_{2}$, which was stained by 3,3'-diaminobenzidine (DAB). The red arrows indicate appressoria formed from conidia. The above photos were taken with a stereo-microscope, scale bars $=1 \mathrm{~mm}$. The photos at the down portion were taken with a microscope (Zeiss imager A2), scale bars $=40 \mu \mathrm{m}$. $\mathbf{b}$ Relative mRNA levels of the defense-related genes (OsKS4, OSNAC4, OsPR1, and OsPR10b) in NPB and OX162 following inoculation of M. oryzae GZ8. Rice ubiquitin $(U B Q)$ gene was served as an internal reference. The mRNA levels were normalized to that in NPB at 0 hpi. Error bars indicate SD $(n=3)$. Different letters above the bars indicate significant differences $(P<0.05)$ as determined by One-way ANOVA analysis. Similar results were obtained in at least two independent experiments

seed setting rate, leading to lower grain number than control (Fig. 6b and Table 1). Substantially, OX162 displayed a slight lower yield per plant (Table 1). In contrast, MIM162 exhibited similar seed size as control (Fig. 6b), slightly lower seed weight (Table 1), lower seed setting rate, but more grains per plant in comparison with control (Fig. $6 \mathrm{c}$ and Table 1). As a result, MIM162 showed higher yield per plant than control (Table 1). These results indicate that OsamiR162a compromises yield, whereas blocking OsamiR162a enhances yield.
OsDCL1 Is Involved in Osa-miR162-Regulated Rice Blast Resistance

Rice Osa-miR162 was reported to target $L O C_{-}$ Os03g02970 (Zhou et al. 2010) and was predicted to target LOC_Os03g15230 (http://plantgrn.noble.org/ psRNATarget/). LOC_OsO3g02970 encodes OsDCL1a, and LOC_OsO3g15230 encodes an expressed protein OsDUF292 (domains of unknown function protein 292; Additional file 3: Figure S3b). We examined the expression of Osa-miR162 and the two genes in 11 independent OX162 lines and 11 independent 


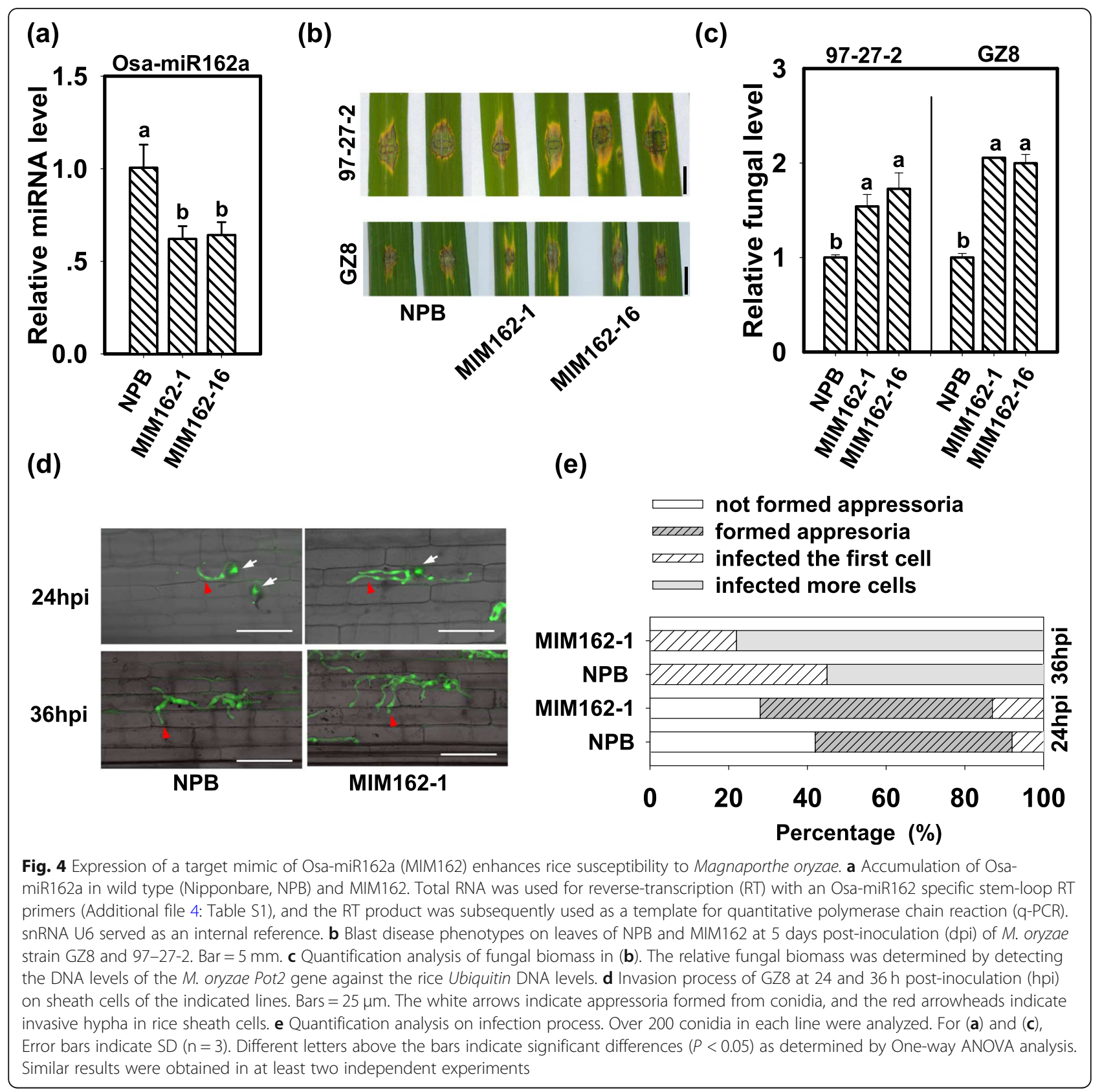

MIM162 lines, respectively. All the 22 detected lines displayed normal gross morphology and produced seeds. Seven of the 11 tested OX162 lines showed significantly higher Osa-miR162 accumulation (Fig. 7a). Conversely, six of the seven lines displayed significant decrease, and the one line showed slight decrease of OsDCL1 mRNA levels (Fig. 7a). Besides, among the 11 tested MIM162 lines, nine lines displayed significantly lower Osa-miR162 abundance in comparison with NPB control (Fig. 7b). Conversely, five of the nine lines displayed significant increase of OsDCL1
mRNA amounts, and three of the nine lines showed slight increase (Fig. 7b). These results indicated the regulation of Osa-miR162a on OsDCL1.

Intriguingly, the mRNA levels of OsDCL1 decreased mildly in all the seven lines overexpressing OsamiR162 (Fig. 7a). Even in the four OX162 lines showing over 40-fold amounts of Osa-miR162a, the mRNA levels of OsDCL1 only decreased less than $50 \%$ of that of control (Fig. 7a), indicating that OsDCL1 is critical for rice normal development, and Osa-miR162 had a limited impact on the expression of OsDCL1 in 
(a)
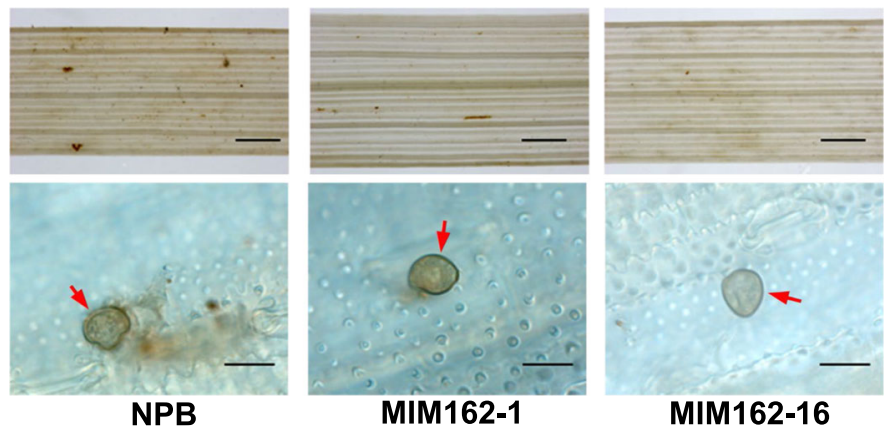

(b)
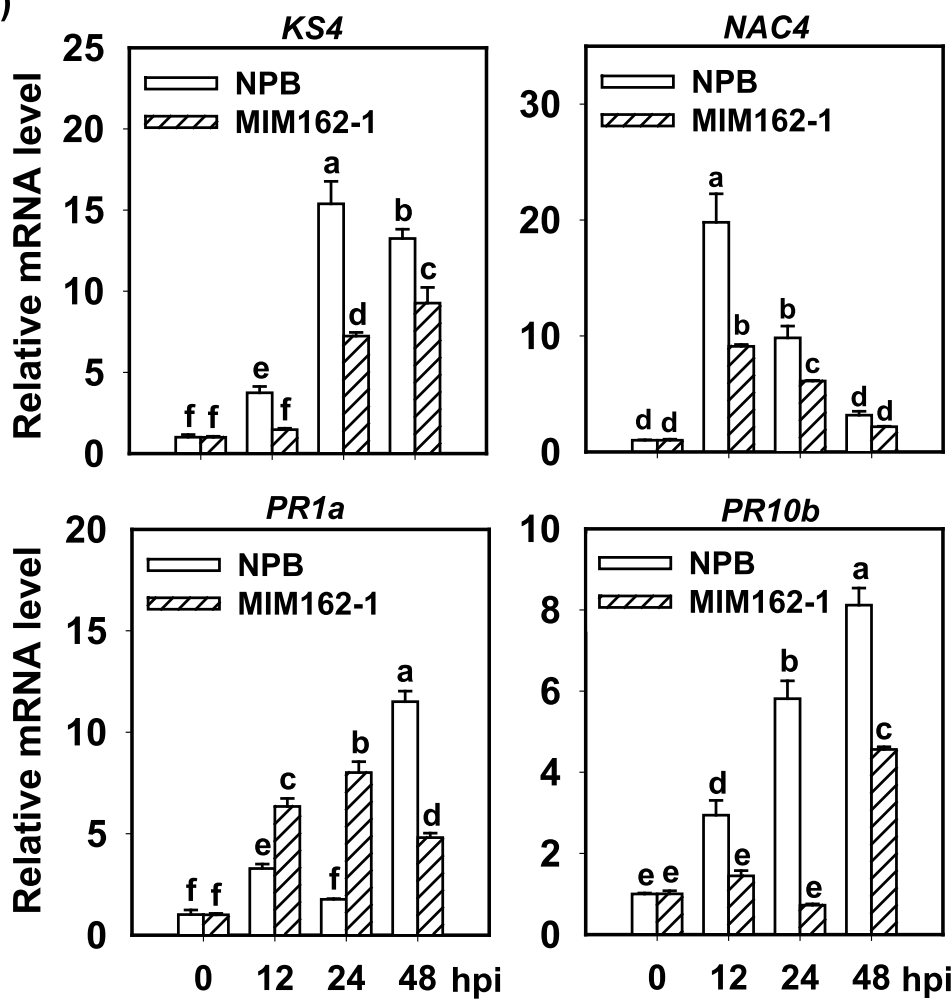

Fig. 5 Blocking Osa-miR162a compromises the induction of disease-related defense responses. a Hydrogen peroxide $\left(\mathrm{H}_{2} \mathrm{O}_{2}\right)$ accumulation in wild type (Nipponbare, NPB) and MIM162 at $48 \mathrm{~h}$ post-inoculation (hpi) of M. oryzae strain GZ8. The intensity of brown indicates the amounts of $\mathrm{H}_{2} \mathrm{O}_{2}$, which was stained by 3,3'-diaminobenzidine (DAB). The red arrows indicate appressoria formed from conidia. The upper photos were taken with a stereo-microscope, scale bars $=1 \mathrm{~mm}$. The photos at the down portion were taken with a microscope (Zeiss imager A2), scale bars $=40 \mu \mathrm{m}$. $\mathbf{b}$ Expression of the defense-related genes (OsKS4, OsNAC4, OsPR1, and OsPR106) in NPB and MIM162 following GZ8 inoculation. Rice ubiquitin (UBQ) gene was served as an internal reference. The mRNA levels were normalized to that in NPB at 0 hpi. Error bars indicate SD $(n=3)$. Different letters above the bars indicate significant differences $(P<0.05)$ as determined by One-way ANOVA analysis. Similar results were obtained in at least two independent experiments

these lines. However, the mRNA levels of OsDUF292 were markedly higher in both OX162 and MIM162 (Additional file 3: Figure S3b), suggesting Osa-miR162 might not directly target OsDUF292.

To explore the roles of OsDCL1 in rice resistance, we examined the expression of OsDCL1 in susceptible accession LTH and resistance accessions IRBLKm-Ts and Yahui2115 upon M. oryzae infection, respectively.
Yahui2115 is an elite hybrid restorer line carrying several blast resistance genes and widely used in breeding programs (Shi et al. 2015). The mRNA amounts of OsDCL1 increased in LTH at 24 and 48 hpi of $M$. oryzae (Fig. 7c), and were reversely consistent with the amounts of Osa-miR162a in LTH following inoculation (Fig. 1b), indicating OsDCL1 was involved in rice resistance to blast fungus and down- 
(a)
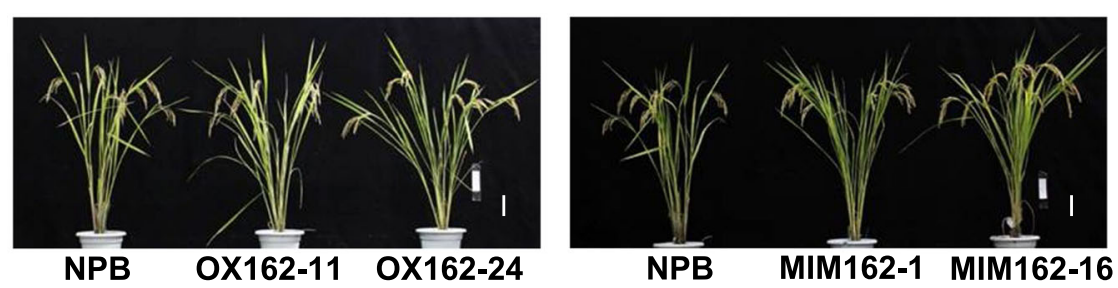

(b)
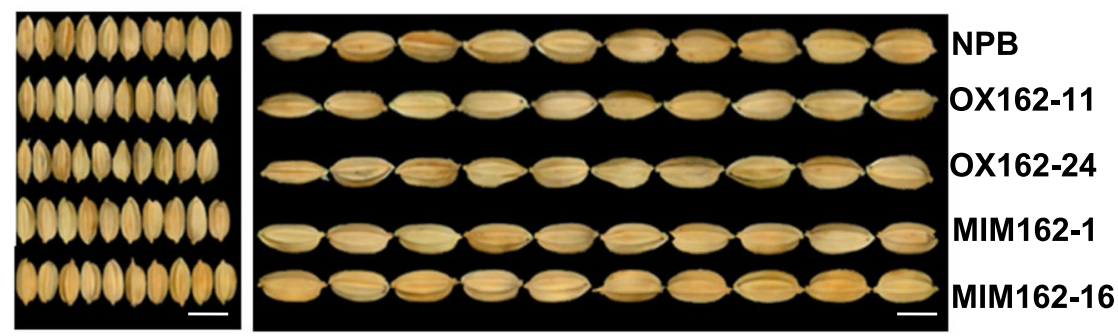

(c)

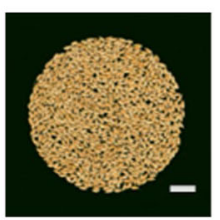

NPB

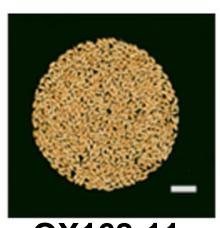

OX162-11

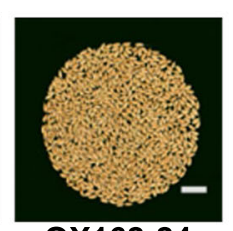

0X162-24

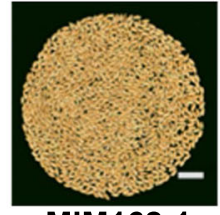

MIM162-1

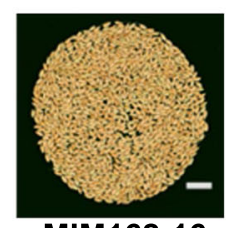

MIM162-16

Fig. 6 Osa-miR162a regulates rice yield. a Gross morphology of the wild type (Nipponbare, NPB), OX162, and MIM162 lines. Scale bars, $10 \mathrm{~cm}$. b Photos of grains to show grain length and grain width of NPB, OX162, and MIM162 lines. Scale bars, 5 mm. c Photo of grains per plant of NPB, OX162, and MIM162 lines. Scale bars, $2 \mathrm{~cm}$

regulated by Osa-miR162a. In contrast, The mRNA amounts of OsDCL1 were decreased in IRBLKm-Ts and Yahui2115 (Fig. 7c), further suggesting the involvement of OsDCL1 in rice resistance to $M$. oryzae. We then examined the roles of OsDCL1 in rice blast resistance by inoculation of GZ8 on an OsDCL1 RNA inference line (DCL1i, (Liu et al. 2005)) showing significantly lower OsDCL1 mRNA amounts than control (Fig. 7d). DCL1i line exhibited enhanced resistance to GZ8 with smaller disease lesions and supported less fungal growth (Fig. 7e and f), which was similar to the disease phenotype of OX162, and was consistent with the previous report that down-regulation of OsDCL1 leads to enhanced resistance to blast fungus (Zhang et al. 2015). Together, these results indicate that OsDCL1 possibly participates in Osa-miR162regulated rice resistance against blast fungus.

\section{Discussion}

MiRNAs play essential roles in controlling plant developmental progress and responses to biotic or abiotic stresses (de Lima et al. 2012; Seo et al. 2013). In recent years, rice- $M$. oryzae interaction has become a model in the study of plant-fungi interaction (Liu and Wang 2016). More than 70 miRNAs were responsive to $M$. ory$z a e$ or its elicitors and 11 of which have been identified as regulators in rice- $M$. oryzae interaction ( $\mathrm{Li}$ et al. 2019b; Zhou et al. 2019). For example, seven miRNAs were identified as negative regulators in rice immunity against $M$. oryzae by disease assays on overexpressing or

Table 1 The agronomic traits of OX162 and MIM162

\begin{tabular}{|c|c|c|c|c|c|c|c|}
\hline Strain & Plant height (cm) & Panicle number & Panicle length (cm) & $\begin{array}{l}\text { Setting Rate } \\
(\%)\end{array}$ & Grain number & $\begin{array}{l}\text { 1000-grain weight } \\
\text { (g) }\end{array}$ & $\begin{array}{l}\text { Yield per plant } \\
\text { (g) }\end{array}$ \\
\hline NPB & $91.4 \pm 2.4^{a}$ & $9.2 \pm 0.44^{a}$ & $19.6 \pm 0.74^{a}$ & $92.7 \pm 0.93^{a}$ & $833 \pm 45^{a}$ & $26.9 \pm 0.63^{a}$ & $22.3 \pm 2.35^{\mathrm{ab}}$ \\
\hline OX-11 & $90.8 \pm 2.16^{a}$ & $9.4 \pm 1.34^{\mathrm{a}}$ & $19.5 \pm 0.63^{a}$ & $87.4 \pm 1.52^{a}$ & $850 \pm 74^{a}$ & $23.4 \pm 0.56^{c}$ & $20.7 \pm 3.90^{b}$ \\
\hline$O X-24$ & $89.6 \pm 2.88^{a}$ & $10.6 \pm 1.5^{a}$ & $19.6 \pm 0.7^{a}$ & $60.7 \pm 7.06^{a}$ & $839 \pm 65^{a}$ & $22.8 \pm 0.38^{c}$ & $18.3 \pm 2.76^{b}$ \\
\hline MIM-1 & $94.8 \pm 4.02^{a}$ & $9.4 \pm 0.89^{a}$ & $19.8 \pm 0.89^{a}$ & $90.5 \pm 3.14^{a}$ & $987 \pm 36^{a}$ & $25.9 \pm 0.38^{a b}$ & $24.1 \pm 3.35^{a}$ \\
\hline MIM-16 & $93.7 \pm 4.4^{\mathrm{a}}$ & $10.4 \pm 0.89^{a}$ & $19.2 \pm 0.94^{\mathrm{a}}$ & $85.9 \pm 0.77^{a}$ & $999 \pm 71^{a}$ & $25.4 \pm 0.3^{b}$ & $24.4 \pm 3.75^{a}$ \\
\hline
\end{tabular}

Notes: Data are shown as mean \pm SD $(n=5)$. Means labeled with different letters indicate a significant difference at $5 \%$ level via Tukey-Kramer test 


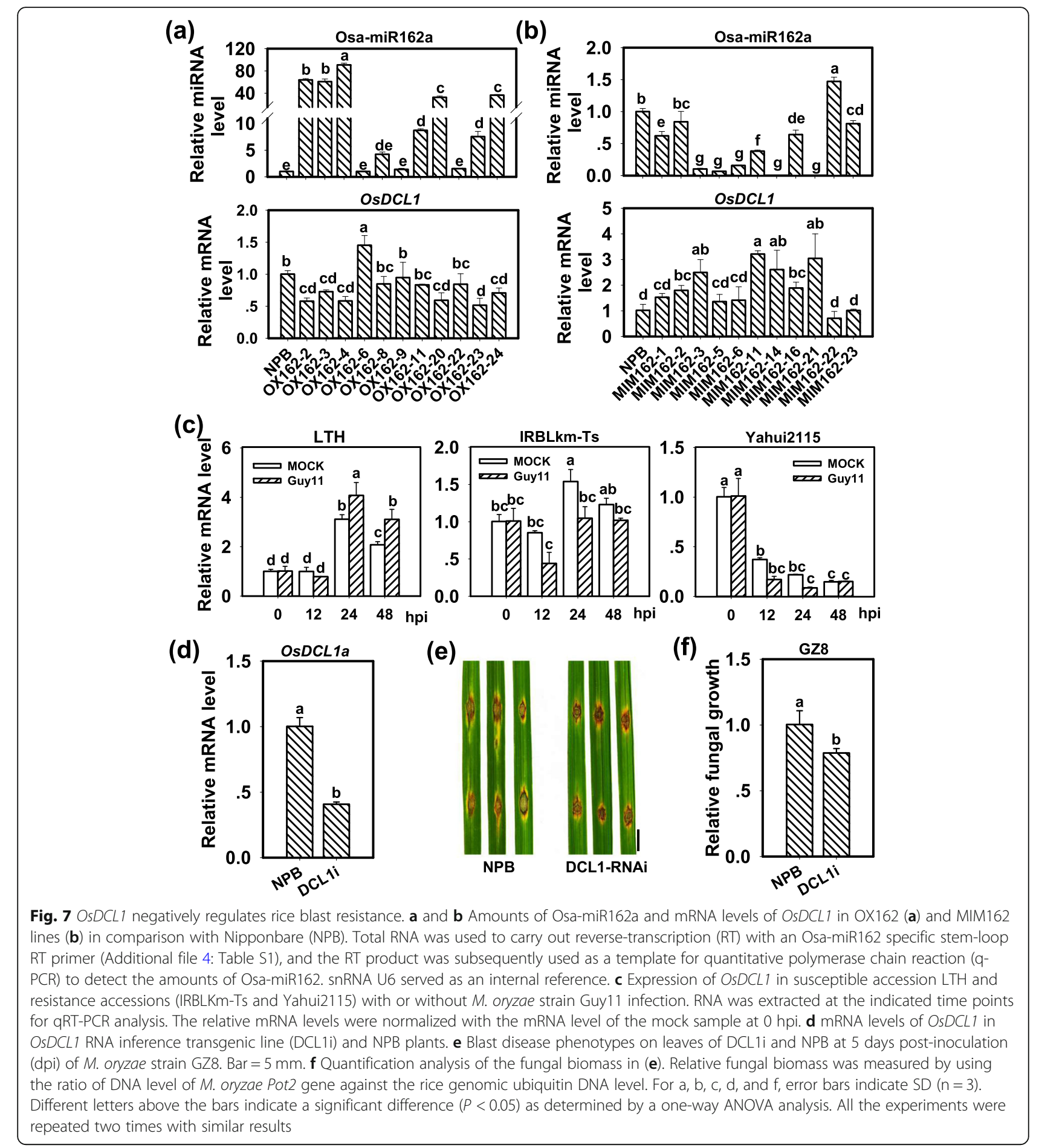

silencing transgenic lines, namely miR164a, miR169a, miR167d, miR319, miR396, miR444, and miR1873 (Li et al. 2017; Xiao et al. 2017; Chandran et al. 2018; Wang et al. 2018; Zhang et al. 2018a; Zhang et al. 2018b; Zhao et al. 2019; Zhou et al. 2019). In contrast, four miRNAs were characterized as positive regulators, namely miR160a, miR166k-miR166h, miR398b, and miR7695
(Campo et al. 2013; Li et al. 2014; Salvador-Guirao et al. 2018; $\mathrm{Li}$ et al. 2019a). Here we characterized OsamiR162a as a positive regulator in rice immunity against M. oryzae. First, the expression of Osa-miR162a was unchanged or decreased in susceptible accession LTH but up-regulated in resistance accession IRBLKm-Ts following M. oryzae infection (Fig. 1). Second, overexpression 
of Osa-miR162a enhanced rice resistance with higher induction of defense responses (Figs. 2 and 3). In contrast, blocking Osa-miR162a by expressing a target mimic compromised rice resistance associating with the suppression of defense responses (Figs. 4 and 5).

Besides the regulation of blast disease resistance, OsamiR162 also fine-tunes rice yield and yield traits. The trade-off between yield and disease resistance is a common phenomenon in crop production. For example, the mutation in SQUAMOSA promoter-binding protein-like transcription factors 28 (OSSPL28) resulted in enhanced resistance to $M$. oryzae but led to a reduction in rice yield (Qiao et al. 2010). miRNAs may act as the crucial fine-tuning regulators to balance rice blast resistance and yield by regulating the expression of their target genes. For example, miR396 fine-tunes rice blast disease resistance and yield by controlling the expression of Growth Regulating Factor (GRF) transcription factor genes. The transgenic lines overexpressing miR396 showed enhanced susceptibility to $M$. oryzae, whereas the transgenic lines blocking miR396 exhibited enhanced rice resistance and increased yield (Chandran et al. 2018). In this study, we demonstrate that Osa-miR162a fine-tunes rice blast resistance and yield. Overexpression of Osa-miR162a leads to enhanced rice blast disease resistance but decreased yield, whereas blocking OsamiR162 results in enhance susceptibility to $M$. oryzae but increased yield, indicating that the accurate accumulation of Osa-miR162a is necessary for the trade-off between yield and resistance.

Osa-miR162a possibly regulates rice-blast fungus interaction via OsDCL1-controlled miRNA network. OsDCL1 RNAi lines displayed blast resistance similar to OX162: higher blast disease resistance (Fig. 7g-h) with increased induction of $\mathrm{H}_{2} \mathrm{O}_{2}$ and cell death at infected sites (Liu et al. 2005; Zhang et al. 2015). In the weak OsDCL1-knocking down mutant, more than ten miRNAs were identified as OsDCL1-regulated miRNAs for their differential expression in OsDCL1 RNAi lines and control (Liu et al. 2005; Zhang et al. 2015). For example, the accumulation of eight miRNAs was abolished or significantly reduced in weak-loss-of-function transformants of OsDCL1, including miR156, miR159, miR166, miR167, miR168, miR168-3p, miR396, and miR528 (Liu et al. 2005). Besides, in another OsDCL1 RNAi lines, the abundance of 12 miRNAs was decreased significantly, including miR156, miR166, miR167, miR168, and miR396 (Zhang et al. 2015). Intriguingly, among these miRNAs, four miRNAs were characterized as regulators in rice- $M$. oryzae interaction, namely miR166, miR167, miR169, and miR396 (Tang and Chu 2017), suggesting that Osa-miR162a possibly regulated rice-blast fungus interaction by controlling the miRNA network via OsDCL1.
Osa-miR162a possibly regulates rice yield traits via impact on OsDCL1, which is essential for rice normal development. OsDCL1 RNAi lines with heavy loss of function showed developmental arrest at the seedling stage, including shoot and root abnormalities at an early developing stage, wilting of leaves and early senescence at a later stage, and eventually died without seed production (Liu et al. 2005), indicating OsDCL1 was essential for rice normal development. The lines with weak loss of function displayed pleiotropic developmental defects but with seed production (Liu et al. 2005). In this study, we found that OX162 lines with seed production all displayed a slightly impact on the expression of OsDCL1. In OX162 lines showing over 40-fold amounts of OsamiR162a, the expression of OsDCL1 decreased less than $50 \%$ of that of control (Fig. 7a), whereas, in MIM162 lines showing significant decreasing of Osa-miR162, the expression of OsDCL1 was increased less than four-fold of that of control (Fig. 7b). We selected two OX162 lines showing an appropriate $10 \%$ to $20 \%$ decrease of OsDCL1 amounts and two MIM162 lines displaying less than a 2fold increase of OsDCL1a mRNA levels in comparison with that of control. The two OX162 lines showed slight defects in yield traits but enhanced blast disease resistance, suggesting that we could control the abundance of Osa-miR162a to manipulate resistance without significant loss of yield.

\section{Conclusions}

Collectively, our results show that Osa-miR162a finetunes rice resistance and yield. Overexpression of OsamiR162a leads to enhanced rice resistance to $M$. oryzae but slightly decreased yield per plant, whereas blocking Osa-miR162 results in enhanced susceptibility but increased yield per plant. Further study reveals that in the OX162 lines we studied, Osa-miR162 had a limited impact on the expression of OsDCL1, and silencing of which leads to similar resistance phenotype as that caused by overexpression of Osa-miR162a, indicating OsDCL1 is involved in miR162-regulated resistance. Thus, Osa-miR162a positively regulates rice blast resistance and can be used to manipulate resistance without great penalty of yield.

\section{Materials and Methods}

\section{Plant Materials and Growth Conditions}

Rice plants used in this study included susceptible accession LTH, resistance accessions IRBLkm-Ts and Yahui2115. Japonica accession NPB was used to construct transgenic lines OX162 or MIM162a, respectively. The transgenic line silencing OsDCL1 is a generous gift from Prof. Xiaofeng Cao (Institute of Genetics and Developmental Biology, Chinese Academy of Sciences). For transformation, fungal inoculation, and defense responses 
assay, rice plants were planted at a growth room at $26^{\circ} \mathrm{C}$ and $70 \%$ relative humidity with $14 / 10$-h of day/night light. For yield traits detection, rice plants were planted in paddy yard at a regular season in Wenjiang district, Sichuan Province, China.

\section{Plasmid Construction and Genetic Transformation}

The transgenic plants were generated following previous protocols ( $\mathrm{Li}$ et al. 2017). To construct the transgenic lines overexpressing Osa-miR162a, the sequence of OsamiR162a gene containing $400 \mathrm{bp}$ upstream and $318 \mathrm{bp}$ downstream sequences was amplified from NPB genomic DNA with primers Osa-miR162a-F and OsamiR162a-R (Additional file 4: Table S1). Then the amplified fragment was cloned into KpnI-SalI sites of binary vector 35S-pCAMBIA1300 and got the overexpression construct p35S: Osa-miR162a. To construct the target mimic of Osa-miR162a, target mimic sequences of OsamiR162a (CTGGATGCAGAAGTGGTTTATCGA) were formed by annealing with primers MIM162a-BamHI-F and MIM162a-BglII-R (Additional file 4: Table S1). The annealing double-strand product was inserted into BamHI- BglII sites of Arabidopsis IPS1 gene to substitute the target site of miR399 as described previously (Franco-Zorrilla et al. 2007; Li et al. 2017). The reconstructed IPS1-MIM162 sequence was cloned into KpnISalI sites of the binary vector pCAMBIA1300, resulting in overexpressing construct p35S: MIM162. The vectors were transformed into NPB by Agrobacterium strain EHA105, and the positive transgenic lines were screened with Hygromycin B.

\section{Pathogen Infection and Microscopy Analysis}

M. oryzae strains Guy11, 97-27-2, GZ8, DZ-6, DZ-31, DZ-46, and DZ-110 were used in this study. 97-27-2 was a strain derived from paddy yard in Sichuan province, China, and GZ8 was a strain derived from a rice field in the north of China tagged with enhanced GFP. DZ-6, DZ-31, DZ-46, and DZ-110 were strains collected from the paddy yard in Dazhu county, Sichuan Province, China. The isolates were cultured in oatmeal/tomato media at $28{ }^{\circ} \mathrm{C}$ with $12-\mathrm{h} / 12$-h light/dark cycles. Two weeks later, the hyphae were scratched, and the plates were continuously cultured at $28^{\circ} \mathrm{C}$ with 24 -h light for sporulation. After 4 days post-inoculation (dpi), spores were collected, and the inoculum concentration was adjusted to $5 \times 10^{5}$ spore $/ \mathrm{ml}$ for punch- and sprayinoculation. T3 seedlings of transgenic plants at threeleaf-stage were used for spray inoculation following previous reports $(\mathrm{Qu}$ et al. 2006). The disease phenotypes on the leaf two were recorded at $5 \mathrm{dpi}$. For punchinoculation, the leaves of T3 seedlings at three-leaf-stage were slightly wounded with a mouse ear punch, and $5 \mu \mathrm{l}$ of spore suspension was drop-inoculated on the wound.
The disease phenotypes were recorded at $5 \mathrm{dpi}$, and the inoculated leaves were collected for fungal biomass assay. The relative fungal biomass was measured using the DNA amounts of fungal Mopot2 against rice ubiquitin DNA amounts by quantitative PCR (Li et al. 2017). For invasive observation, the 5-cm-long leaf sheaths of the seedlings at five-leaf-stage were inoculated with spores suspension of eGFP-tagged $M$. oryzae strain GZ8 $\left(2 \times 10^{5} / \mathrm{ml}\right)$ as described previously (Kankanala et al. 2007). The epidermal layer was excised for observation at 24 and $36 \mathrm{~h}$ post-inoculation (hpi). The fungal growth process was analyzed by Laser Scanning Confocal Microscopy (Nikon A1), and the infestation stage was analyzed as described previously ( $\mathrm{Li}$ et al. 2014). For $\mathrm{H}_{2} \mathrm{O}_{2}$ detection, three-leaf-stage seedlings were spray inoculated with GZ8 $\left(5 \times 10^{5} / \mathrm{ml}\right)$. The leaves were collected at $36 \mathrm{hpi}$ and placed in 3, 3'-Diaminobenzidine (DAB) (1 mg /ml, Sigma, ALORICH, USA) for dying. The dying leaves were shook at room temperature for $12 \mathrm{~h}$ in a dark place, and then decolorized in $90 \%$ ethyl alcohol at $65{ }^{\circ} \mathrm{C}$ for several times (Xiao et al. 2003). The photo showing $\mathrm{H}_{2} \mathrm{O}_{2}$ accumulation on leaves was captured with a stereomicroscope (Zeiss imager). $\mathrm{H}_{2} \mathrm{O}_{2}$ accumulation in leaf cells and fungal structures were observed with a microscope (Zeiss imager A2).

\section{RNA Extraction and Quantitative Reverse-Transcription Polymerase Chain Reaction (qRT-PCR) Analysis}

Three-leaf-stage plants were inoculated with or without $M$. oryzae by spraying spore suspensions at a concentration of $5 \times 10^{5} / \mathrm{ml}$, and the leaves were collected at $0,12,24$, and 48 hpi. Total RNA was extracted from these leaves using Trizol reagent (Invitrogen) and was used to detect the amounts of Osa-miR162 and gene expression. The qRTPCR analysis was used to detect the amounts of OsamiR162a and expression of genes. The RNA quality and quantity were determined by a spectrophotometer (NanoDrop2000 Uvevis). For analysis of amounts of Osa-miR162, Stem-loop pulse reverse-transcription was performed using the qPCR-RT Master Mix with gDNA Remover (TOYOBO) with Osa-miR162a stem-loop primer (Additional file 4: Table S1) following the direction. Then the RT products were used as templates for qPCR with the primers Osa-miR162a-RT-F and universal reverse primer (Additional file 4: Table S1) to detect the amounts of OsamiR162 (Chen et al. 2005; Varkonyi-Gasic et al. 2007). For analysis of the expression of genes, the first-strand cDNA was synthesized using the qPCR-RT Master Mix with gDNA Remover (TOYOBO) following the direction. Then the expression of genes was detected with specific forward and reverse primers listed in Additional file 4: Table S1. U6 snRNA was used as an internal reference to normalize Osa-miR162 levels (Turner et al. 2013), and the rice ubiquitin $(U B Q)$ gene was selected as an internal reference of 
the detected genes. qRT-PCR was performed using SYBR Green mix (Quanti Nova SYBR Green PCR Kit, QIGEN, Chengdu, China) with BIO-RAD C1000TM Thermal Cycler (Bio-Rad Inc., Chengdu, China).

\section{Yield Trait Measurements}

NPB, OX162, and MIM162 lines were planted in paddy yard at a regular season in 2019 at Wenjiang District, Sichuan Province, China. Plant height, panicle number per plant, panicle length, seed setting rate, grain number per panicle, 1000-grain weight, and yield per plant were measured at full maturity from five plants. The filled grains were dried in a $42^{\circ} \mathrm{C}$ oven for 1 week. The 1000 -grain weight was measured using an SC-A grain analysis system (Wanshen Ltd., Hangzhou, China). All the data of yield traits were analyzed by One-Way ANOVA followed by post hoc Tukey HSD analysis at a significant level of 0.05 .

\section{Supplementary information}

Supplementary information accompanies this paper at https://doi.org/10. 1186/s12284-020-00396-2.

\begin{abstract}
Additional file 1: Figure S1. Overexpression of Osa-miR162a leads to enhanced resistance to Magnaporthe oryzae upon spray-inoculation. (a) Disease phenotypes on leaves of the indicated lines following sprayinoculation with M. oryzae strain GZ8. The phenotype was captured at 5 days post-inoculation. Bar $=5 \mathrm{~mm}$. (b) Quantification analysis of the fungal biomass in (a). Relative fungal biomass was measured by using the ratio of DNA level of M. oryzae Pot2 gene against the rice genomic ubiquitin DNA level. Error bars indicate SD $(n=3)$. Different letters above the bars indicate a significant difference $(P<0.05)$ as determined by a one-way ANOVA analysis. Similar results were obtained in at least two independent experiments.
\end{abstract}

Additional file 2: Figure S2. Overexpression of Osa-miR162a results in enhanced resistance to multiple Magnaporthe oryzae strains. (a) Disease phenotypes on leaves of NPB, OX162 and MIM162 following punchinoculation with field-derived $M$. oryzae strains. The phenotype was captured at 5 days post-inoculation. Bars $=5 \mathrm{~mm}$. (b-e) Quantification analysis of the fungal biomass in (a). Relative fungal biomass was measured by using the ratio of DNA level of $M$. oryzae Pot2 gene against the rice genomic ubiquitin DNA level. Error bars indicate SD $(n=3)$. Different letters above the bars indicate a significant difference $(P<0.05)$ as determined by a one-way ANOVA analysis. Similar results were obtained in at least two independent experiments

Additional file 3: Figure S3. Osa-miR162a does not repress the expression of DUF292. (a) The alignment of Osa-miR162a and Osa-miR162b with MIM162. (b) The sequence alignment of Osa-miR162a with target sites of candidate genes. Mismatched nucleotides were highlighted in red colors. (c and d) Quantitative reverse transcription polymerase chain reaction (RT-qPCR) data indicate amounts of Osa-miR162a levels in OX162 (c) and MIM162 lines (d) in comparison with Nipponbare (NPB). For qPCR, Data are shown as mean $\pm S D(n=3)$. Different letters indicate a significant difference at $5 \%$ level via Tukey-Kramer test. All the experiments were repeated two times with similar results.

Additional file 4: Table S1. Primers used in this study.

\section{Abbreviations}

M. oryzae: Magnaporthe oryzae; miRNAs: MicroRNAs; OsDCL1: Dicer-like 1; $\mathrm{H}_{2} \mathrm{O}_{2}$ : Hydrogen peroxide; nt: Nucleotides; AGO: ARGONAUTE; miRISC: MiRNA-Induced Silence Complex; OSTRE1: Trehalase precursor 1; LTH: Lijiangxin Tuan Heigu; R: Resistance; OX162: Transgenic lines overexpressing Osa-miR162a; GZ8: GFP-tagged Zhong 8-10-14; KS4: ENT-KA
RENE SYNTHASE 4; NAC4: NAC DOMAIN-CONTAINING PROTEIN 4; PR1a: PATHOGENESIS-RELATE GENG 1A; MIM162: The transgenic lines overexpressing a target mimic of Osa-miR162a; OsDUF292: Domains of unknown function protein; DCL1i: OsDCL1 RNA inference line;

OsSPL28: SQUAMOSA promoter-binding protein-like transcription factors 28; GRF: Growth Regulating Factor

\section{Acknowledgments}

We thank Dr. Cai-Lin Lei (Institute of Crop Science, Chinese Academy of Agricultural Sciences) for providing the monogenic resistant lines IRBLkm-Ts, Dr. Xiao-Feng Cao (Institute of Genetics and Development, Chinese Academy of Sciences) for providing OsDCL1 RNAi transgenic lines.

\section{Authors' Contributions}

$Y L$ and $W W$ designed the research; $X L, X M, H W, Y Z$, and $X L$ conducted the experiments; TL, YZ, JZ, JZ, YH, MP, HF, and JF analyzed the data; YL wrote the article; $W W$ supervised and complemented the manuscript. The authors read and approved the manuscript.

\section{Funding}

This work was supported by the National Natural Science Foundation of China (No. 31430072, 31672090 and U19A2033 to WW).

\section{Availability of Data and Materials}

The data sets supporting the conclusions of this article are included within the article and its additional files.

Ethics Approval and Consent to Participate

Not applicable.

\section{Consent for Publication}

Not applicable.

\section{Competing Interests}

The authors declare that they have no competing interests

\section{Author details}

${ }^{1}$ State Key Laboratory of Crop Gene Exploration and Utilization in Southwest China, Sichuan Agricultural University, Chengdu, China. ${ }^{2}$ Rice Research Institute and Key Lab for Major Crop Diseases, Sichuan Agricultural University, Chengdu, China.

Received: 30 January 2020 Accepted: 27 May 2020

Published online: 10 June 2020

\section{References}

Campo S, Peris-Peris C, Sire C, Moreno AB, Donaire L, Zytnicki M, Notredame C, Llave C, Segundo BS (2013) Identification of a novel microRNA (miRNA) from rice that targets an alternatively spliced transcript of the Nramp6 (natural resistance-associated macrophage protein 6) gene involved in pathogen resistance. New Phytol 199:212-217

Chandran V, Wang H, Gao F, Cao XL, Chen YP, Li GB, Zhu Y, Yang XM, Zhang LL, Zhao ZX, Zhao JH, Wang YG, Li S, Fan J, Li Y, Zhao JQ, Li SQ, Wang WM (2018) miR396-OsGRFs module balances growth and Rice blast diseaseresistance. Front. Plant Sci 9:1999

Chen C, Ridzon DA, Broomer AJ, Zhou Z, Guegler KJ (2005) Real-time quantification of microRNAs by stem-loop RT-PCR. Nucleic Acids Res 33: e179

de Lima JC, Loss-Morais G, Margis R (2012) MicroRNAs play critical roles during plant development and in response to abiotic stresses. Genet Mol Biol 35: 1069-1077

Ding D, Zhang L, Wang H, Liu Z, Zhang Z, Zheng Y (2009) Differential expression of miRNAs in response to salt stress in maize roots. Ann Bot 103:29-38

Franco-Zorrilla JM, Valli A, Todesco M, Mateos I, Puga MI, Rubio-Somoza I, Leyva A, Weigel D, Garcia JA, Paz-Ares J (2007) Target mimicry provides a new mechanism for regulation of microRNA activity. Nat Genet 39:1033-1037

Kankanala P. Czymmek K, Valent B (2007) Roles for rice membrane dynamics and plasmodesmata during biotrophic invasion by the blast fungus. Plant Cell 19:706-724

Katiyar-Agarwal S, Jin H (2010) Role of small RNAs in host-microbe interactions. Annu Rev Phytopathol 48:225-246 
Kurihara Y, Watanabe Y (2004) Arabidopsis micro-RNA biogenesis through dicerlike 1 protein functions. Proc Natl Acad Sci U S A 101:12753-12758

Li Y, Cao XL, Zhu Y, Yang XM, Zhang KN, Xiao ZY, Wang H, Zhao JH, Zhang LL, Li GB, Zheng YP, Fan J, Wang J, Chen XQ, Wu XJ, Zhao JQ, Dong OX, Chen XW, Chern M, Wang WM (2019a) Osa-miR398b boosts H2 O2 production and rice blast disease-resistance via multiple superoxide dismutases. New Phytol. https://doi.org/10.1111/nph.15678

Li Y, Jeyakumar JMJ, Feng Q, Zhao ZX, Fan J, Khaskheli MK, Wang WM (2019b) The roles of rice microRNAs in rice-Magnaporthe oryzae interaction. Phytopathology Research Published: 18 November 2019. 1:33

Li Y, Lu YG, Shi Y, Wu L, Xu YJ, Huang F, Guo XY, Zhang Y, Fan J, Zhao JQ, Zhang HY, Xu PZ, Zhou JM, Wu XJ, Wang PR, Wang WM (2014) Multiple rice microRNAs are involved in immunity against the blast fungus Magnaporthe oryzae. Plant Physiol 164:1077-1092

Li Y, Zhao SL, Li JL, Hu XH, Wang H, Cao XL, Xu YJ, Zhao ZX, Xiao ZY, Yang N, Fan J, Huang F, Wang WM (2017) Osa-miR169 negatively regulates Rice immunity against the blast fungus Magnaporthe oryzae. Front Plant Sci 8:2

Lin ZZ, Jiang WW, Wang JL, Lei CL (2001) Research and utilization of universally susceptible property of japonica rice variety Lijiangxintuanheigu. Scientia Agricultura Sinjca 34:116-117

Liu B, Li P, Li X, Liu C, Cao S, Chu C, Cao X (2005) Loss of function of OsDCL1 affects microRNA accumulation and causes developmental defects in rice. Plant Physiol 139:296-305

Liu W, Wang GL (2016) Plant innate immunity in rice: a defense against pathogen infection. Natl Sci Rev 3:295-308

Navarro L, Dunoyer P, Jay F, Arnold B, Dharmasiri N, Estelle M, Voinnet O, Jones JD (2006) A plant miRNA contributes to antibacterial resistance by repressing auxin signaling. Science 312:436-439

Park CH, Chen S, Shirsekar G, Zhou B, Khang CH, Songkumarn P, Afzal AJ, Ning Y, Wang R, Bellizzi M, Valent B, Wang GL (2012) The Magnaporthe oryzae effector AvrPiz-t targets the RING E3 ubiquitin ligase APIP6 to suppress pathogenassociated molecular pattern-triggered immunity in Rice. Plant Cell 24:4748-4762

Park W, Li J, Song R, Messing J, Chen X (2002) CARPEL FACTORY, a dicer homolog, and HEN1, a novel protein, act in microRNA metabolism in Arabidopsis thaliana. Curr Biol 12:1484-1495

Pertermann R, Tamilarasan S, Gursinsky T, Gambino G, Schuck J, Weinholdt C, Lilie H, Grosse I, Golbik RP, Pantaleo V, Behrens SE (2018) A viral suppressor modulates the plant immune response early in infection by regulating MicroRNA activity. mBio 9. https://doi.org/10.1128/mBio.00419-18

Qiao Y, Jiang W, Lee J, Park B, Choi MS, Piao R, Woo MO, Roh JH, Han L, Paek NC, Seo HS, Koh HJ (2010) SPL28 encodes a clathrin-associated adaptor protein complex 1, medium subunit micro 1 (AP1M1) and is responsible for spotted leaf and early senescence in rice (Oryza sativa). New Phytol 185:258-274

Qu S, Guifu L, Bo Z, Maria B, Lirong Z, Liangying D, Bin H, Guo-Liang W (2006) The broad-spectrum blast resistance gene Pi9 encodes a nucleotide-binding site-leucine-rich repeat protein and is a member of a multigene family in rice. Genetics 172:1901-1914

Salih H, Gong W, Mkulama M, Du X (2018) Genome-wide characterization, identification, and expression analysis of the WD40 protein family in cotton. Genome 61:539-547

Salvador-Guirao R, Baldrich P, Tomiyama S, Hsing Yl, Okada K, San Segundo B (2019) OsDCL1a activation impairs phytoalexin biosynthesis and compromises disease resistance in rice. Ann Bot 123:79-93

Salvador-Guirao R, Hsing YI, San Segundo B (2018) The Polycistronic miR166k$166 \mathrm{~h}$ positively regulates Rice immunity via post-transcriptional control of EIN2. Front Plant Sci 9:337

Seo JK, Wu J, Lii Y, Li Y, Jin H (2013) Contribution of small RNA pathway components in plant immunity. Mol Plant Microbe Interact 26:617-625

Shi J, Li D, Li Y, Li X, Guo X, Luo Y, Lu Y, Zhang Q, Xu Y, Fan J, Huang F, Wang W (2015) Identification of rice blast resistance genes in the elite hybrid rice restorer line Yahui2115. Genome 58:91-97

Sun G, Stewart CN Jr, Xiao P, Zhang B (2012) MicroRNA expression analysis in the cellulosic biofuel crop switchgrass (Panicum virgatum) under abiotic stress. PLoS One 7:e32017

Tang J, Chu C (2017) MicroRNAs in crop improvement: fine-tuners for complex traits. Nat Plants 3:17077

Tian C, Zuo Z, Qiu JL (2015) Identification and characterization of ABA-responsive MicroRNAs in Rice. J Genet Genomics 42:393-402

Tijsterman M, Plasterk RH (2004) Dicers at RISC; the mechanism of RNAi. Cell 117: $1-3$
Tsumematsu H, Yanoria MJT, Ebron LA, Hayashi N, Ando I, Kato H, Imbe T, Khush GS (2000) Development of monogenic lines of rice for blast resistance. Breed Sci 50:229-234

Turner M, Adhikari S, Subramanian S (2013) Optimizing stem-loop qPCR assays through multiplexed cDNA synthesis of U6 and miRNAs. Plant Signal Behav 8:e24918

Varkonyi-Gasic E, Wu R, Wood M, Walton EF, Hellens RP (2007) Protocol: a highly sensitive RT-PCR method for detection and quantification of microRNAs. Plant Methods 3:12

Wang Z, Xia Y, Lin S, Wang Y, Guo B, Song X, Ding S, Zheng L, Feng R, Chen S, Bao Y, Sheng C, Zhang X, Wu J, Niu D, Jin H, Zhao H (2018) Osa-miR164a targets OsNAC60 and negatively regulates rice immunity against the blast fungus Magnaporthe oryzae. Plant J. https://doi.org/10.1111/tpj.13972

Weiberg A, Wang M, Bellinger M, Jin H (2014) Small RNAs: a new paradigm in plant-microbe interactions. Annu Rev Phytopathol 52:495-516

Wu L, Zhang Q, Zhou H, Ni F, Wu X, Qi Y (2009) Rice MicroRNA effector complexes and targets. Plant Cell 21:3421-3435

Xiao S, Brown S, Patrick E, Brearley C, Turner JG (2003) Enhanced transcription of the Arabidopsis disease resistance genes RPW8.1 and RPW8.2 via a salicylic acid-dependent amplification circuit is required for hypersensitive cell death. Plant Cell 15:33-45

Xiao ZY, Wang WQ, Zhao SL, Wang H, Li JL, Huang F, Fan J, Li Y, Wang WM (2017) MiR444b.2 regulates resistance to Magnaporthe oryzae and tillering in rice. Acta Phytopathol Sin 47:511-522

Xie Z, Johansen LK, Gustafson AM, Kasschau KD, Lellis AD, Zilberman D, Jacobsen SE, Carrington JC (2004) Genetic and functional diversification of small RNA pathways in plants. PLoS Biol 2:E104

Yamaguchi K, Yamada K, Ishikawa K, Yoshimura S, Hayashi N, Uchihashi K, Ishihama N, Kishi-Kaboshi M, Takahashi A, Tsuge S, Ochiai H, Tada Y, Shimamoto K, Yoshioka H, Kawasaki T (2013) A receptor-like cytoplasmic kinase targeted by a plant pathogen effector is directly phosphorylated by the chitin receptor and mediates Rice immunity. Cell Host Microbe 13:347357

Yin Z, Chen J, Zeng L, Goh M, Leung H, Khush GS, Wang GL (2000) Characterizing rice lesion mimic mutants and identifying a mutant with broad-spectrum resistance to rice blast and bacterial blight. Mol Plant Microbe Interact 13:869-876

Yu Y, Jia T, Chen X (2017) The 'how' and 'where' of plant microRNAs. New Phytol 216:1002-1017

Zhang D, Liu M, Tang M, Dong B, Wu D, Zhang Z, Zhou B (2015) Repression of microRNA biogenesis by silencing of OsDCL1 activates the basal resistance to Magnaporthe oryzae in rice. Plant Sci 237:24-32

Zhang X, Bao Y, Shan D, Wang Z, Song X, Wang J, He L, Wu L, Zhang Z, Niu D, Jin H, Zhao H (2018a) Magnaporthe oryzae induces the expression of a MicroRNA to suppress the immune response in Rice. Plant Physiol 177:352-368

Zhang X, Bao Y, Shan D, Wang Z, Song X, Wang Z, Wang J, He L, Wu L, Zhang Z (2018b) Magnaporthe oryzae defeats rice defense by inducing miR319b and suppressing Jasmonic acid signaling. Plant Physiol 177:01665.02017

Zhao ZX, Feng Q, Cao XL, Zhu Y, Wang H, Chandran V, Fan J, Zhao JQ, Pu M, Li Y, Wang WM (2019) Osa-miR167d facilitates infection of Magnaporthe oryzae in rice. J Integr Plant Biol. https://doi.org/10.1111/jipb.12816

Zhou M, Gu LF, Li PC, Song XW, Wei LY, Chen ZY, Cao XF (2010) Degradome sequencing reveals endogenous small RNAtargets in rice (Oryza sativa L. ssp. indica). Front Biol 5:67-90

Zhou SX, Zhu Y, Wang LF, Zheng YP, Chen JF, Li TT, Yang XM, Wang H, Li XP, Ma $X C$, Zhao JQ, Pu M, Feng H, Li Y, Fan J, Zhang JW, Huang YY, Wang WM (2019) Osa-miR1873 fine-tunes rice immunity against Magnaporthe oryzae and yield traits. J Integr Plant Biol. https://doi.org/10.1111/jipb.12900

\section{Publisher's Note}

Springer Nature remains neutral with regard to jurisdictional claims in published maps and institutional affiliations. 